\title{
Robust Treatment of Simultaneous Collisions
}

\author{
David Harmon \\ Columbia University \\ Etienne Vouga \\ Columbia University \\ Rasmus Tamstorf
Walt Disney Animation Studios \\ Eitan Grinspun \\ Columbia University
}
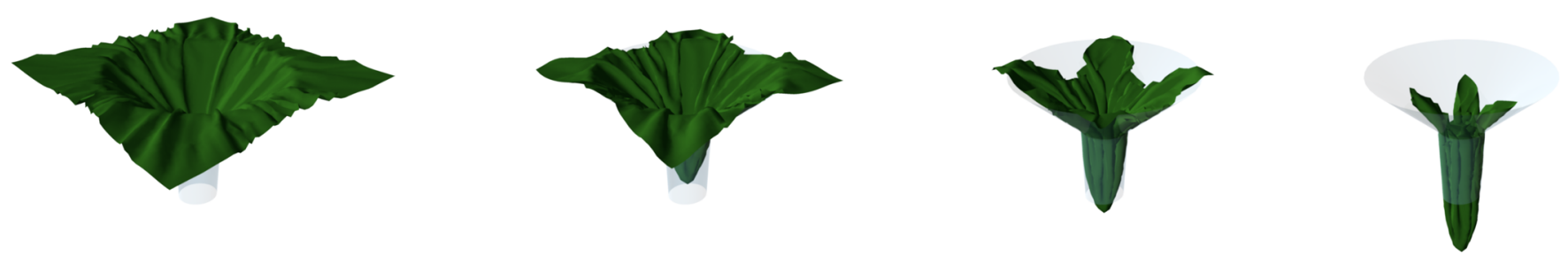

Figure 1: Stress test. Shoving cloth through a narrow funnel yields free-flowing motion, despite the complex network of contact regions.

\begin{abstract}
Robust treatment of complex collisions is a challenging problem in cloth simulation. Some state of the art methods resolve collisions iteratively, invoking a fail-safe when a bound on iteration count is exceeded. The best-known fail-safe rigidifies the contact region, causing simulation artifacts. We present a fail-safe that cancels impact but not sliding motion, considerably reducing artificial dissipation. We equip the proposed fail-safe with an approximation of Coulomb friction, allowing finer control of sliding dissipation.
\end{abstract}

CR Categories: I.3.7 [Computer Graphics]: Three-Dimensional Graphics and Realism-Animation

Keywords: cloth, simulation, shells, collision, contact, configuration space

\section{Introduction}

Robust collision response is a vital research area in cloth and shell simulation; not only must collisions be prevented, but the response must be physical. While the handling of individual collisions is well understood, simultaneous collisions can halt existing methods.

The response framework of Bridson et al. [2002] is widely adopted in industry for its efficiency, versatility, and proven ability to solve practical problems. Its efficiency is partly due to a built-in failsafe: when facing a cluster of interacting simultaneous collisions, the framework extracts the rigid body motion of the cluster, as presented by Provot [1997]. This rigid impact zone (RIZ) approach prevents collisions, but also eliminates all relative tangential velocity, unphysically dissipating energy from the system. In a simple simulation, a RIZ is rarely needed; however, many complex and practically-relevant scenes require frequent invocations of the failsafe, which leads to excessive energy dissipation and even unphysical locking of otherwise free-flowing fabric. To partially alleviate this rigidification, Huh et al. [2001] divided the impact zone into clusters, and Tsiknis [2006] considered shearing modes.
We present a simple projection method for resolving simultaneous collisions, while preserving as much tangential motion as possible given the time step resolution. This method serves as an improved fail-safe for the framework of Bridson et al. [2002], increasing the quality, consistency, and physical plausibility of simulations, as we demonstrate in a series of challenging example scenarios designed to exercise the fail-safe. In addition, we show that the method is compatible with an approximation of Coulomb friction, allowing the choice of any desired sliding dissipation.

\section{Theory}

Configuration space The entire mesh, at any instant in time, can be viewed as a point in a high-dimensional space; for an $n$-vertex mesh with vertices $X_{1}, X_{2}, \ldots, X_{n} \in \mathbb{R}^{3}$, the configuration space is $\mathbf{Q}=\mathbb{R}^{3 n}$. As the mesh evolves through time, it traces out the curve $\left[X_{1}(t), \ldots, X_{n}(t)\right]^{T}=\mathbf{q}(t) \in \mathbf{Q}$ (a column vector by convention). Using a dot to denote differentiation with respect to time, $\dot{\mathbf{q}}(t)$ is the configurational velocity at any instant $t$ [Lanczos 1986]. Lastly, $\mathbf{M}$ is the mass matrix, $\mathbf{p}(t)=\mathbf{M} \dot{\mathbf{q}}(t)$ is the momentum, and $\frac{1}{2}\langle\mathbf{p}, \mathbf{p}\rangle_{\mathbf{M}^{-1}}=\frac{1}{2} \mathbf{p}^{T} \mathbf{M}^{-1} \mathbf{p}$ is the kinetic energy.

Collision Constraints We view each collision as the violation of a real-valued constraint function, $C(\mathbf{q})$, with $C(\mathbf{q})<0$ whenever $\mathbf{q}$ is an inadmissable (penetrating) configuration. We associate one constraint function to each impending vertex-triangle or edge-edge collision. The constraint for an impending collision between the triangle $\left(X_{1}(t), X_{2}(t), X_{3}(t)\right)$ and the vertex $X_{4}(t)$ is

$$
C_{\mathrm{vt}}(\mathbf{q})=N \cdot\left[X_{4}-\left(\alpha_{1} X_{1}+\alpha_{2} X_{2}+\alpha_{3} X_{3}\right)\right],
$$

where $N(t)$ is the triangle normal, and the three scalars $\alpha_{1}(t), \alpha_{2}(t)$, and $\alpha_{3}(t)$ are the barycentric coordinates of the projection of $X_{4}(t)$ onto the plane spanned by the triangle. Similarly, the constraint for an impending collision between the edges $\left(X_{1}(t), X_{2}(t)\right)$ and $\left(X_{3}(t), X_{4}(t)\right)$ is

$$
C_{\mathrm{ee}}(\mathbf{q})=N \cdot\left[\left(\alpha_{3} X_{3}+\alpha_{4} X_{4}\right)-\left(\alpha_{1} X_{1}+\alpha_{2} X_{2}\right)\right],
$$

where $\alpha_{1}(t), \alpha_{2}(t), \alpha_{3}(t)$ and $\alpha_{4}(t)$ are the parametric values of corresponding closest points on the first and second edge, respectively, and $N(t)$ is the cross product of the two edges. If the two edges are parallel at the time of contact we ignore it, since this collision will be detected by the vertex-triangle constraint. We refer to the rate of change of the constraint function, $\dot{C}$, as the normal velocity. By convention, $N(t)$ is oriented such that a negative normal velocity corresponds to approaching primitives. 
Unlike collision detection and response algorithms that solve these constraints as a function of time [Snyder et al. 1993], we formulate them primarily to reason about and resolve collisions in configuration space. While each constraint depends on only four vertices (called the stencil), it is formally a scalar function of $\mathbf{q}$, and can be differentiated with respect to $\mathbf{q}$ to yield the constraint gradient, $\nabla C$, a row vector in configuration space. By the chain rule, we may rewrite the normal velocity as $\dot{C}=\nabla C \dot{\mathbf{q}}$. For a vertextriangle collision, the constraint gradient expressed in the local indices of the stencil is

$$
\nabla C_{\mathrm{vt}}=\left(-\alpha_{1} N,-\alpha_{2} N,-\alpha_{3} N, N\right) ;
$$

for an edge-edge collision, the gradient in local indices is

$$
\nabla C_{\mathrm{ee}}=\left(-\alpha_{1} N,-\alpha_{2} N, \alpha_{3} N, \alpha_{4} N\right) .
$$

To elevate the constraint gradient to configuration space, we simply map the local indices to their global positions, with zeros everywhere else, in the style of finite element stiffness matrix assembly.

Impulse response for a single constraint Let unprimed and primed quantities refer to the pre- and post-response states, respectively. A valid response satisfies two properties. First, it pushes colliding objects apart by applying a nonnegative impulse along the constraint direction, i.e., $\mathbf{p}-\mathbf{p}^{\prime}=\nabla C^{T} \lambda$ for some (unknown) nonnegative multiplier $\lambda$. Second, since the impulse should prevent colliding points from approaching further, it must lead to a nonnegative post-response normal velocity $\nabla C \dot{\mathbf{q}}^{\prime}=\nabla C \mathrm{M}^{-1} \mathbf{p}^{\prime}$.

For an inelastic collision, we seek the maximally dissipative response among all valid responses, per the definition of purely inelastic. Thus, we minimize post-response kinetic energy, $\frac{1}{2}\left\|\mathbf{p}^{\prime}\right\|_{\mathrm{M}^{-1}}^{2}=\frac{1}{2}\left\|\mathbf{p}+\nabla C^{T} \lambda\right\|_{\mathrm{M}^{-1}}^{2}$, with respect to $\lambda$, which yields

$$
\lambda=\frac{-\nabla C \dot{\mathbf{q}}}{\langle\nabla C, \nabla C\rangle_{\mathrm{M}^{-1}}} .
$$

One property of the above inelastic response is that the normal velocity vanishes along the constraint direction: $\nabla C \dot{\mathbf{q}}^{\prime}=0$.

Impulse response for multiple constraints Cirak and West [2005] treat the above case of a single constraint in detail. We, however, are interested in the case of $k$ simultaneous constraints, i.e., $k$ vertex-triangle and edge-edge collisions with possibly nondisjoint stencils. Now let $\nabla C=\left[\nabla C_{1}^{T}, \ldots, \nabla C_{k}^{T}\right]^{T}$ be a $k \times 3 n$ matrix whose rows span the possible impulse directions. For any vector $\boldsymbol{\lambda} \in \mathbb{R}^{k}, \mathbf{p}^{\prime}=\mathbf{p}+\nabla C^{T} \boldsymbol{\lambda}$ corresponds to the application of a linear combination of collision impulses to $\mathbf{p}$. As in the single-constraint case, we require that the $\lambda_{1} \ldots \lambda_{k}$ be nonnegative, since constraint impulses can push but not pull, and that the impulse yields nonnegative post-response relative velocities, i.e., that every row of $\nabla C \dot{\mathbf{q}}^{\prime}=\left[\nabla C_{1} \dot{\mathbf{q}}^{\prime}, \ldots, \nabla C_{k} \dot{\mathbf{q}}^{\prime}\right]^{T}$ be nonnegative.

Solving for the $\lambda$ that minimizes kinetic energy, subject to the above constraints on $\boldsymbol{\lambda}$ and normal velocity, can be formulated as a linear complementarity problem (LCP), solvable using methods such as those described by Baraff [1994]. For multiple collisions with overlapping stencils, some response impulses may be redundant, i.e., responding to a subset of all the collisions may satisfy all collision constraints. The LCP approach ensures that redundant collisions do not yield pulling impulses $\left(\lambda_{i}<0\right)$.

However, since black-box linear solvers are more readily available than LCP solvers, we propose an algorithm for approximating $\boldsymbol{\lambda}$ by assuming that post-response relative velocities are exactly zero: $\nabla C \dot{\mathbf{q}}^{\prime}=0$. This may result in some $\lambda_{i}<0$, introducing artificial

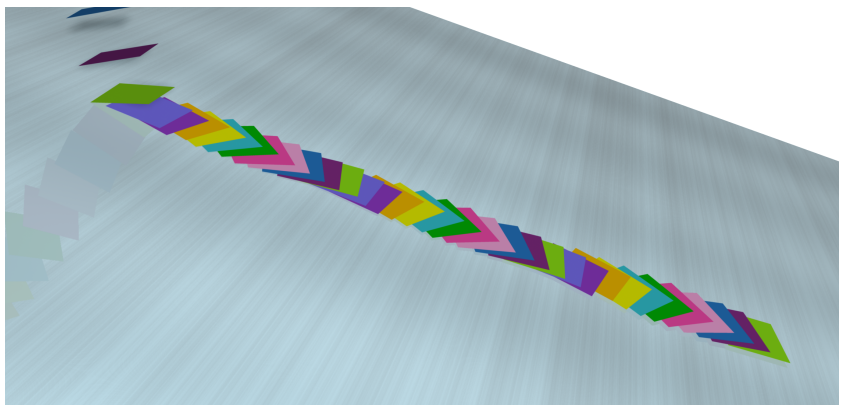

Figure 2: Stacking. Our projection method easily handles a multitude of simultaneous collisions with overlapping stencils, allowing each piece of stacked fabric to freely flow down the inclined plane.

"sticking." Observe that our simplification affects only the fail-safe; since Bridson et al.'s framework resorts to the fail-safe only after many iterations of repulsive impulses that are designed to prevent sticking, we have not observed any significant sticking artifacts.

If we relax the conditions on a response being valid to allow both positive and negative entries in $\lambda$, then $\dot{\mathbf{q}}^{\prime}$ is the minimizer of

$$
\left\|\dot{\mathbf{q}}-\dot{\mathbf{q}}^{\prime}\right\|_{M}^{2}, \quad \text { subject to } \nabla C \dot{\mathbf{q}}^{\prime}=\mathbf{0} .
$$

This minimization projects the velocity onto the orthogonal complement of the span of the columns of $\nabla C^{T}$. Hence we call this the inelastic projection. We may repose the above as an extremization of the augmented functional

$$
W\left(\dot{\mathbf{q}}^{\prime}, \boldsymbol{\lambda}\right)=\frac{1}{2}\left\|\dot{\mathbf{q}}^{\prime}-\dot{\mathbf{q}}\right\|_{M}^{2}+\left(\nabla C \dot{\mathbf{q}}^{\prime}\right)^{T} \boldsymbol{\lambda}
$$

with respect to $\left(\dot{\mathbf{q}}^{\prime}, \boldsymbol{\lambda}\right)$, where $\boldsymbol{\lambda}$ is a vector of Lagrange multipliers. The corresponding stationary equations are

$$
\begin{aligned}
& 0=D_{1} W\left(\dot{\mathbf{q}}^{\prime}, \boldsymbol{\lambda}\right)=\mathbf{p}^{\prime}-\mathbf{p}+\nabla C^{T} \boldsymbol{\lambda} \\
& 0=D_{2} W\left(\dot{\mathbf{q}}^{\prime}, \boldsymbol{\lambda}\right)=\nabla C \dot{\mathbf{q}}^{\prime}
\end{aligned}
$$

Equation (3) guarantees that the response acts only along the $\nabla C$ direction, and (4) ensures vanishing normal velocities. Substituting (3) into (4), it follows that we can recover the inelastic response for a set of $k$ simultaneous collisions by solving the linear system

$$
\nabla C M^{-1} \nabla C^{T} \boldsymbol{\lambda}=\nabla C \dot{\mathbf{q}}
$$

for $\boldsymbol{\lambda}$, and then substituting it into (3) to obtain the unique postinelastic-collision momentum, $\mathbf{p}^{\prime}$.

In theory, the length of the gradient vectors does not matter, but we normalize the constraint gradients to improve numerical robustness. Even so, when collision detection creates a set of nearly parallel gradients, the above linear system can be poorly conditioned or even singular. Thus we recommend the use of an iterative solver intended for near-singular systems when solving (5).

\section{Algorithm}

Our method is a replacement step in the algorithm of Bridson et al. [2002], which is divided into three stages of response:

- repulsion forces,

- continuous collision response (CCR), and

- rigid impact zones (RIZ). 
Our method replaces the RIZ step with an inelastic projection step. In the case of a single collision, our method reduces to the CCR step, so it is acceptable to replace the CCR step as well. When the colliding region grows, our method expands in the same manner as the RIZ step (see Algorithm 1).

Each constraint gradient depends on a stencil of four vertices (recall $\S 2$ ). We define an impact zone (IZ) as a set of constraint gradients with overlapping stencils. Inelastic projection is performed per IZ, and since vertices outside of the IZ are unaffected by collision response, we restrict $\mathbf{q}$ in $\S 2$ to be the subset of configuration space corresponding to the vertices in the impact zone. Furthermore, the gradients we use are evaluated at the time of collision.

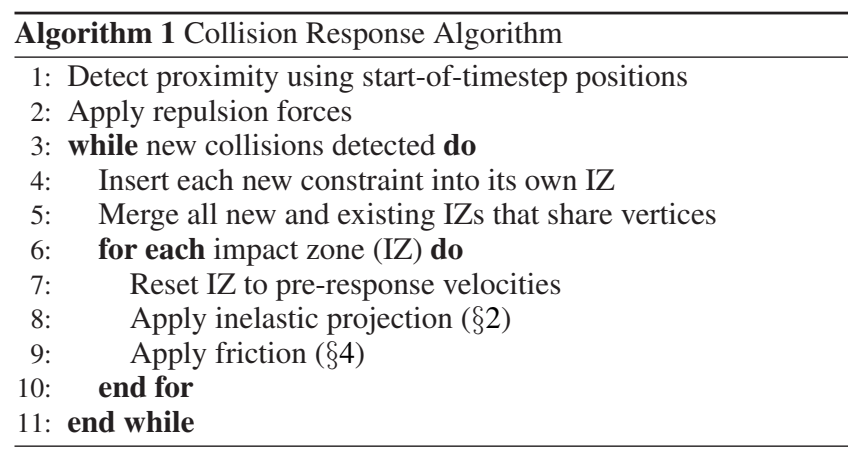

One may resolve cloth-object and self-collision in separate passes as mentioned by Bridson et al. [2003]. However, the passes must be prioritized, potentially allowing penetrations in the lower-priority pass. To prevent unresolved collisions in the two-pass approach, Algorithm 1 responds to all self-collisions and cloth-object collisions simultaneously. Our collision step treats prescribed objects and simulated cloth alike; the infinite mass of prescribed objects ensures that they are unaffected by simulated cloth.

Scripted objects with infinite mass proved difficult for RIZs. Any RIZ that contains a scripted (cloth or object) vertex has its motion, in part, determined by that vertex. If a RIZ contains multiple vertices whose velocities are not consistent with a unique rigid motion, then the RIZ fails and the timestep must be halved (since in general smaller timesteps lead to smaller RIZs). On the other hand, inelastic projection finds a solution unless the vertices are constrained in fundamentally opposed directions, e.g., a vertex is being pinched by scripted objects.

After we apply a normal impulse to stop an impending collision, we are free to apply a corresponding frictional impulse. In $\S 4$, we propose a simple friction model that is guaranteed not to introduce new collisions within the IZ. Nevertheless, the frictional impulse applied to the IZ may cause new collisions with vertices outside the IZ. If this occurs, we grow the IZ to include the new vertices (Algorithm 1, line 5), reset the IZ to pre-collision velocities (line 7), and restart the process.

\section{Friction}

The complex interaction between collisions requires the careful selection of the direction in which friction is applied, lest more collisions be instigated. We apply friction per IZ by again restricting q to be the configuration subspace corresponding to the vertices of the IZ. Vertices outside the impact zone remain untouched.
Building on Cirak and West [2005], we decompose the configurational velocity as

$$
\dot{\mathbf{q}}^{\prime}=\dot{\mathbf{q}}_{\text {fix }}^{\prime}+\dot{\mathbf{q}}_{\text {norm }}^{\prime}+\dot{\mathbf{q}}_{\text {slide }}^{\prime} .
$$

These three global velocity vectors are characterized by the local velocities they induce on each constraint: $\dot{\mathbf{q}}_{\text {fix }}^{\prime}$ corresponds to zero relative velocity (normal and tangential) for all constraints; $\dot{\mathbf{q}}_{\text {norm }}^{\prime}$ corresponds to purely normal velocity for all constraints; $\dot{\mathbf{q}}_{\text {slide }}^{\prime}$ is the sliding velocity for which friction must be applied.

Following inelastic projection, $\dot{\mathbf{q}}_{\text {norm }}^{\prime}$ is zero. With that, we have

$$
\dot{\mathbf{q}}_{\text {slide }}^{\prime}=\dot{\mathbf{q}}^{\prime}-\dot{\mathbf{q}}_{\text {fix }}^{\prime} .
$$

To find $\dot{\mathbf{q}}_{\text {fix }}^{\prime}$, we project out motion that induces a relative velocity for any constraint. For a single constraint, this relative velocity is

$$
V_{\text {rel }}=\nabla h \dot{\mathbf{q}}^{\prime},
$$

where $h(\mathbf{q})=X_{4}-\left(\alpha_{1} X_{1}+\alpha_{2} X_{2}+\alpha_{3} X_{3}\right)$ for a vertex-triangle collision and $h(\mathbf{q})=\left(\alpha_{3} X_{3}+\alpha_{4} X_{4}\right)-\left(\alpha_{1} X_{1}+\alpha_{2} X_{2}\right)$ for an edgeedge collision, using the same notation as in $\S 2$. For multiple constraints, we project out any velocity in the row space of $\nabla H=\left[\nabla h_{1}^{T}, \ldots, \nabla h_{k}^{T}\right]^{T}$, where $\nabla H$ is a $3 k \times 3 n$ matrix and $k$ is the number of constraints in the IZ, leaving us with $\dot{\mathbf{q}}_{\mathrm{fix}}^{\prime}$. To implement this projection, observe that $\dot{\mathbf{q}}_{\mathrm{fix}}^{\prime}$ is the minimizer of

$$
\left\|\dot{\mathbf{q}}^{\prime}-\dot{\mathbf{q}}_{\mathrm{fix}}^{\prime}\right\|_{M}^{2}, \quad \text { subject to } \nabla H \dot{\mathbf{q}}_{\mathrm{fix}}^{\prime}=\mathbf{0} .
$$

We solve the linear system $\nabla H \mathrm{M}^{-1} \nabla H^{T} \boldsymbol{\lambda}^{\prime}=\nabla H \dot{\mathbf{q}}$ for $\boldsymbol{\lambda}^{\prime} \in \mathbb{R}^{3 n}$ to recover $\dot{\mathbf{q}}_{\mathrm{fix}}^{\prime}=\mathbf{q}^{\prime}+\mathrm{M}^{-1} \nabla H^{T} \boldsymbol{\lambda}^{\prime}$ and $\dot{\mathbf{q}}_{\text {slide }}^{\prime}$ (see (6)).

From the sliding velocity $\dot{\mathbf{q}}_{\text {slide }}^{\prime}$, we can apply an approximation of Coulomb friction. We would like the friction to be proportional to the normal force, which is simply the change in relative velocity, bounded by the pre-collision sliding velocity. Given a preprojection velocity $\dot{\mathbf{q}}$, post-projection velocity $\dot{\mathbf{q}}^{\prime}$, and friction coefficient $\mu$, the change in velocity due to friction is

$$
\Delta \dot{\mathbf{q}}=\min \left(\mu\left\|\dot{\mathbf{q}}^{\prime}-\dot{\mathbf{q}}\right\|,\left\|\dot{\mathbf{q}}_{\text {slide }}^{\prime}\right\|\right) \widehat{\dot{\mathbf{q}}_{\text {slide }}^{\prime}} .
$$

Note that the amount of friction applied is dictated by the magnitude of the normal force in configuration space. Thus a large normal force for one constraint may induce a larger friction impulse on other constraints. This operation is performed per impact zone, so in practice the colliding region is small and local enough that this approximation yields plausible results. A correct treatment could build on the work of Kaufman et al. [2005], who intersect with a limit curve for each constraint, preventing extraneous friction. However, for the purposes of a fail-safe, our experiments demonstrate that this approximation is satisfactory.

\section{Results}

We tested our method on several challenging scenarios that exercise two possible fail-safes - the original RIZ and inelastic projection.

Figure 1 (and accompanying video) shows frames from our simulation of square cloth being plunged into a small funnel by a scripted ball. This example highlights the importance of allowing freeflowing tangential motion, since otherwise the cloth rigidifies and halts inside the chute. Unlike the inelastic projection, the RIZ failsafe fails to give a plausible response. We also ran this example using two passes of collisions response, as discussed in $\S 3$. Again RIZ fails, this time by allowing the cloth to penetrate the funnel, even with an object thickness 100 times larger than the cloth thickness, and a timestep of $10^{-4}$ seconds. 


\begin{tabular}{|c|r|r|r|r|r|r|}
\hline Example & DOFs & Time Step & Frames & \multicolumn{1}{c|}{ Total } & \multicolumn{1}{c|}{ Collision } & \multicolumn{1}{c|}{ Mean } \\
\hline Ribbon & 7416 & $1 / 480$ & 1000 & 7627.33 & 6647.97 & 6.65 \\
& & & & 5029.29 & 4049.09 & 4.05 \\
\hline Funnel & 16569 & $1 / 480$ & 1000 & 23711.75 & 18814.60 & 18.81 \\
& & & & 13124.61 & 9613.79 & 9.61 \\
\hline Funnel (low-res) & 7695 & $1 / 480$ & 1000 & 18763.28 & 15933.07 & 15.93 \\
& & & & 13714.11 & 10899.00 & 10.90 \\
\hline Crushed Cylinder & 3099 & $1 / 384$ & 1000 & 2833.33 & 1911.35 & 1.91 \\
& & & & 2846.09 & 1923.50 & 1.92 \\
\hline Squares on Incline & 732 & $1 / 24$ & 2500 & 1578.13 & 1540.98 & 0.62 \\
& & & & 853.67 & 816.08 & 0.33 \\
\hline Squares on Plane & 732 & $1 / 24$ & 2500 & 1224.55 & 1178.21 & 0.47 \\
& & & & 1388.75 & 1341.87 & 0.54 \\
\hline
\end{tabular}

Table 1: Performance evaluation. Timing (in seconds) for examples executed on a single thread of a $2.66 \mathrm{Ghz}$ Intel Core 2 Duo with 4GB RAM, comparing RIZ (shaded rows) and inelastic projection (white rows).

Figure 2 (and accompanying video) shows a scenario where tangential sliding is key and RIZ has a fundamental limitation. In this example, we drop a sequence of small (2-triangle) squares onto an incline. Observe how the projection method allows the squares to smoothly slide despite the long chain of simultaneous, interacting collisions. We repeat this example with friction, to reinforce that free-flowing tangential sliding still allows control of friction. As an example where RIZ has an advantage over inelastic projection, we drop a large number of cloth squares in a stack on a flat plane; here the rigid response is physically correct, and linear projection is wasted work.

In the video we show a thin ribbon falling through a trough under varying coefficients of friction. Notice that the linear projection fail-safe gives markedly different behaviors as friction is increased, whereas with RIZ even the frictionless ribbon sticks.

Our algorithm applies to thin shells as well as cloth, as we demonstrate by crushing a plastic cylinder between a thin wire and a narrow crevice.

Table 1 lists timings for many of the above examples. Notice that simulations run with the inelastic projection fail-safe have run times comparable to the RIZ fail-safe, with linear projection at worst $15 \%$ slower than RIZ. Indeed, since most simulation time is spent performing collision detection, and not collision response, the additional linear solve in the proposed fail-safe does not substantially decrease the speed of simulation.

Limitations Our method suffers from all the usual limitations of impulse-based collision response. Additionally, treating all collisions occurring over a timestep as simultaneous may introduce artificial dissipation: if collisions were handled instead in causal order, responding to earlier collisions could prevent subsequent ones.

The linear system solved during inelastic projection can be illconditioned or singular; since it is not acceptable for the fail-safe itself to fail, a numerical method suited for ill-conditioned matrices (such as SVD or GMRES) is strongly recommended. We also tested QLP factorization [Stewart 1999], but unfortunately it did not approximate the singular values well enough for our needs.

Our method has two friction-related limitations. The magnitude of friction is governed by the magnitude of the collision response impulse in configuration space. This magnitude dictates the amount of friction for an entire IZ, potentially resulting in large collision impulses increasing the applied friction for another part of the IZ. Our method also supports only one coefficient of friction per IZ.
Conclusion Since inelastic projection requires a straightforward linear solve, we hope that it can be quickly incorporated into existing frameworks. Our method is compatible with an approximation of Coulomb friction that does not create additional collisions; this allows for the efficient simulation of a wide range of materials, including cloth and thin shells.

We are intrigued by the possibility of incorporating LCP as a failsafe. Thus, we plan to release a technical report outlining this approach with comparisons against the inelastic projection.

Dealing with the shortcomings that our method resolves left us with a new set of exciting research opportunities. In particular, current deformable simulations ignore the causality of collisions, treating any that occur within a timestep as simultaneous. While rigid body simulations can treat collisions in order by stepping to the collision time, resolving it, and starting with new initial conditions, the large number of degrees of freedom in cloth simulation have thus far prohibited such methods. This motivates our ongoing investigation of asynchronous methods for resolving collisions in causal order.

Acknowledgements We would like to thank Mike King for assistance with early renderings of the stacking sequence and Kori Valz for her help with final renderings. This work was supported in part by the NSF (MSPA Award No. IIS-05-28402, CSR Award No. CNS-06-14770, CAREER Award No. CCF-06-43268), Adobe, Autodesk, Elsevier, mental images, and NVIDIA.

\section{References}

BARAFF, D. 1994. Fast contact force computation for nonpenetrating rigid bodies. In SIGGRAPH '94, 23-34.

Bridson, R., Fedkiw, R., And Anderson, J. 2002. Robust treatment of collisions, contact and friction for cloth animation. In SIGGRAPH' 02, 594-603.

Bridson, R., MARino, S., ANd Fedkiw, R. 2003. Simulation of clothing with folds and wrinkles. In SCA '03, 28-36.

Cirak, F., And West, M. 2005. Decomposition-based contact response (DCR) for explicit finite element dynamics. IJNME 64, $8,1078-1110$.

Huh, S., Metaxas, D., AND Badler, N. 2001. Collision resolutions in cloth simulation. In Proceedings of Computer Animation, 122-127.

Kaufman, D. M., Edmunds, T., And PaI, D. K. 2005. Fast frictional dynamics for rigid bodies. In SIGGRAPH '05, 946956.

Lanczos, C. 1986. The Variational Principles of Mechanics, 4th ed. Dover.

Provot, X. 1997. Collision and self-collision handling in cloth model dedicated to design garments. In Computer Animation and Simulation '97, Springer Verlag, Wien, 177-189.

Snyder, J. M., Woodbury, A. R., Fleischer, K., Currin, B., AND BARR, A. H. 1993. Interval methods for multi-point collisions between time-dependent curved surfaces. In $S I G$ GRAPH' '93, 321-334.

Stewart, G. W. 1999. The QLP approximation to the singular value decomposition. SIAM J. Sci. Comput. 20, 4, 1336-1348.

TsIKNIS, K. D. 2006. Better Cloth Through Unbiased Strain Limiting and Physics-Aware Subdivision. Master's thesis, UBC. 